\title{
Lift Force at Equatorial Sea Level Due to Compressed Air Dynamics of the Trade Wind's Boundary Layer
}

\section{Kern E. Kenyon}

4632 North Lane, Del Mar, USA

Correspondence to: Kern E. Kenyon, kernken@aol.com

Keywords: Equatorial Lift Force, Trade Winds, Ocean Heat Balances

Received: April 16, $2021 \quad$ Accepted: June 1, $2021 \quad$ Published: June 4, 2021

Copyright $\odot 2021$ by author(s) and Scientific Research Publishing Inc.

This work is licensed under the Creative Commons Attribution International License (CC BY 4.0).

http://creativecommons.org/licenses/by/4.0/

\section{(c) (1) Open Access}

\section{ABSTRACT}

Starting with a recent unconventional explanation of the lift force on a wing, featuring compressibility of the air, an application of the same concept is made to the lift force on the equatorial sea surface due to the Trade Winds, by greatly increasing the spatial scales. If the equatorial sea level does rise up, the northward slope to the sea level should facilitate the poleward flux of summer heat in both the North Pacific and North Atlantic Oceans, as two examples, in accordance with the heat budget requirements of these oceans. Compressed air dynamics consists of Bernoulli's law applied to the streamlines of the Trade Winds, the force balance between the upward centrifugal force of the curved streamlines at the earth's surface and a downward pressure force, and the perfect gas law for air.

\section{INTRODUCTION}

A novel way of understanding the lift force on a low speed wing [1] is extrapolated to a much larger scale phenomenon: the effect of the Trade Winds on the equatorial ocean surface waters. In both cases, the compressibility of the air is the critical characteristic of the modeling. Also an analogous application has been made to the lift force on the crests of surface gravity waves propagating in the wind which makes them grow [2].

First, there appears to be no reason why Bernoulli's law cannot be applied to the Trade Winds, the steadiest winds on earth [3]. Second, air is compressible so the perfect gas law can be used. Third, streamlines of the Trade Winds are curved around the ocean's surface implying the existence of an upward centrifugal force on flowing air particles which must be balanced by a downward pressure force to maintain steady motion. At this point, there are three differential equations in three unknowns: pressure, density and velocity.

An essential part of the physics and chemistry takes place when the air from the Trade Winds impinges on the incompressible ocean surface, then there forms, it is proposed, a boundary layer of compressed air with greater density than in the environment. 


\section{MODELING}

For a single governing equation in one unknown, pressure $p$, the derivation in [1] is selected to display.

$$
r \frac{\mathrm{d} p}{\mathrm{~d} r}+2 p=0
$$

where $r$ is now the radius from the center of the earth. The solution to (1) is

$$
p=\frac{\text { const }}{r^{2}}
$$

where the constant in (2)is positive but its magnitude needs to be evaluated.

Even before that happens it is clear from (2), by taking the derivative with respect to $r$, that there is an upward pressure force at the earth's surface. Also a fluid will respond to the least amount of force, so equatorial sea levels should rise significantly in response to the lift force, unlike any relatively unyielding land in the region.

If there really is caused an elevated sea level at and near the equator, it would be helpful in carrying out the heat balances of the oceans, as explained below.

\section{DISCUSSION}

In the problem of the lift force on a low speed circular arc wing the functional form of the pressure solution is also a constant times the inverse square of $r$, where $r$ there is measured from the center of the circle of which the arc surface of the wing is a part, where

$$
\text { const }=\rho_{0} h r_{0} S
$$

And $h$ is the maximum thickness of the wing, $r_{0}$ is the radius of the circular arc's top surface and $\rho_{0}$ is the constant environmental air density away from the wing. Also $S=R T$ and $R$ is the gas constant for air and $T$ is the air temperature taken constant.

To adapt the parameters in (3) to a lens shaped segment of an equatorial ocean is easily done for most of them, like $R, \rho_{0}$ and $r_{0}$, which is now the radius from the earth's center to the equatorial sea level. Then $h$ could be taken to be the maximum thickness of the lens. If $T$ should be assumed constant in an open question.

How would a rise in equatorial sea level help the heat balance of the North Atlantic and North Pacific Oceans, for example? Sufficient data to answer this question have been available for well over 50 years, but the following realization based on them has only very gradually come into focus $[4,5]$. In the western tropics of both oceans is a large body of surface water where the highest temperatures can always be found. Not only that, but amazingly the highest temperatures are no higher in summer than in winter! What happens in summer is that the increasing volumes of warm surface waters in both oceans, due to increasing absorption of solar radiation in that season, expand to the north. A rise is sea level at and near the equator would obviously aid such a northward drift of warm surface water and therefore heat.

A different type of lifting force of equatorial sea level has been proposed before [5] based on vertical thermal expansion due to absorbed solar radiation in the top $100 \mathrm{~m}$ of the water column. This mechanism is definitely seasonal because it is most prominent in summer, perhaps in contrast to the lift force of the steady trade winds. What is not known at present is the relative strengths of the two independent methods of lifting up the sea surface.

Lastly it has been known for three hundred years that the equatorial radius of the earth is significantly larger than the polar radius, probably because of the daily rotation of the earth about it axis. This fact results in poleward downward slopes in equatorial sea level as well, and as the surface waters warm up in spring and summer, consequently they start to slide downhill toward the poles. 


\section{CONCLUSION}

A mechanism is proposed for elevating equatorial sea level based on a lift force generated by the Trade Winds. Earlier a different elevation mechanism was suggested to be due to thermal expansion caused by absorbed solar radiation. Either method or both together will help ensure that a poleward transport of heat takes place in the surface layers of the oceans, which is part of the earth's overall heat budget requirement.

\section{CONFLICTS OF INTEREST}

The author declares no conflicts of interest regarding the publication of this paper.

\section{REFERENCES}

1. Kenyon, K.E. (2021) Lift on a Low Speed Circular Arc Wing Due to Air Compression. Natural Science, 13, 88-90. https://doi.org/10.4236/ns.2021.133008

2. Kenyon, K.E. (2021) Wind Wave Growth. Natural Science, 13, 137-139. https://doi.org/10.4236/ns.2021.135013

3. Krishnamurti, T.N., Stefanova, L. and Mirsa, V. (2013) Tropical Meteorology. Springer, New York. https://doi.org/10.1007/978-1-4614-7409-8

4. Kenyon, K.E. (2018) North Pacific's Very Wide Poleward Surface Flow in Summer. Natural Science, 10, 313-317. https://doi.org/10.4236/ns.2018.108031

5. Kenyon, K.E. (2020) North Atlantic's SSTs. Natural Science, 12, 317-319.

https://doi.org/10.4236/ns.2020.126028 